NIST Technical Note 2108

\title{
Towards Measurement of Advanced Mobile Manipulator Performance for Assembly Applications
}

\author{
Roger Bostelman \\ Ya-Shian Li-Baboud \\ Soocheol Yoon \\ Mili Shah \\ Omar Aboul-Enein
}


NIST Technical Note 2108

\title{
Towards Measurement of Advanced Mobile Manipulator Performance for Assembly Applications
}

\author{
Roger Bostelman \\ Smart HLPR, LLC \\ Ya-Shian Li-Baboud \\ Soocheol Yoon \\ Omar Aboul-Enein \\ National Institute of Standards and Technology \\ Mili Shah \\ Department of Mathematics \\ The Cooper Union for the Advancement of Science and Art
}

This publication is available free of charge from:

https://doi.org/10.6028/NIST.TN.2108

August 2020

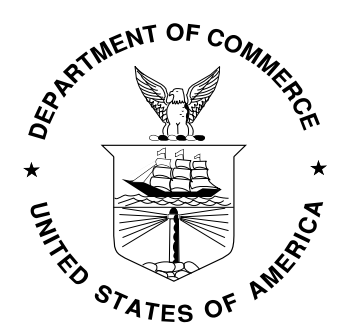

U.S. Department of Commerce

Wilbur L. Ross, Jr., Secretary

National Institute of Standards and Technology Walter Copan, NIST Director and Undersecretary of Commerce for Standards and Technology 
Certain commercial entities, equipment, or materials may be identified in this document in order to describe an experimental procedure or concept adequately. Such identification is not intended to imply recommendation or endorsement by the National Institute of Standards and Technology, nor is it intended to imply that the entities, materials, or equipment are necessarily the best available for the purpose.

The opinions, recommendations, findings, and conclusions in this publication do not necessarily reflect the views or policies of NIST or the United States

Government.

National Institute of Standards and Technology Technical Note 2108

Natl. Inst. Stand. Technol. Tech. Note 2108, 31 pages (August 2020)

CODEN: NTNOEF

This publication is available free of charge from: https://doi.org/10.6028/NIST.TN.2108 


\begin{abstract}
Advanced mobile manipulators offer the manufacturing industry the potential of a highly adaptive system to perform precision assembly tasks in agile environments. Developing performance measurement capabilities for mobile manipulator systems will support the advancement of manipulator-vehicle coordination, precision, accuracy, and robustness. The National Institute of Standards and Technology (NIST) developed a measurement methodology for advanced mobile manipulators, which are mobile robots with an onboard robot arm, each from a different manufacturer and with a different controller. The measurement methodology is designed to assess the performance of mobile manipulators for manufacturing applications such as assembly, where position and orientation accuracy are critically important. The proposed methodology used an artifact designed at NIST to simulate an assembly task station. An optical tracking system measured the location of the targets, the mobile base position and orientation, and the position of a manipulator end-of-arm tool. The experimental data collected, using three different system computers, were timestamped using local clocks synchronized via the NIST internal and local area networks to align the data streams. The mobile manipulator system, the experimental methodology, the data analysis, and the results of the measurement methodology uncertainty and mobile manipulator positioning uncertainty using the novel artifacts are described in this paper. For the system tested, the mobile manipulator detection of $2 \mathrm{~mm}$ diameter fiducials can be achieved after registration. With validation from the optical tracking system, the study demonstrated that the Reconfigurable Mobile Manipulator Artifact (RMMA) has potential for serving as a novel standalone, low-cost test method for measuring the positioning uncertainty of mobile manipulators within a measurement uncertainty of $2 \mathrm{~mm}$.
\end{abstract}

\title{
Key words
}

Mobile robot; robot arm; mobile manipulator; optical tracking system; reconfigurable mobile manipulator artifact (RMMA); ground truth. 


\section{Table of Contents}

1. Introduction ........................................................................................................................... 1

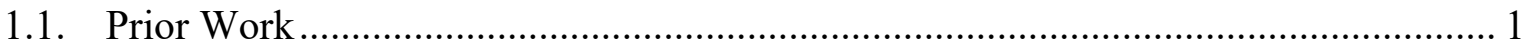

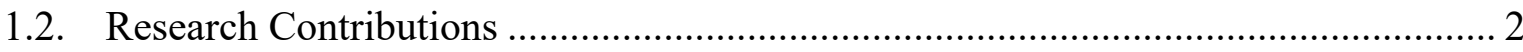

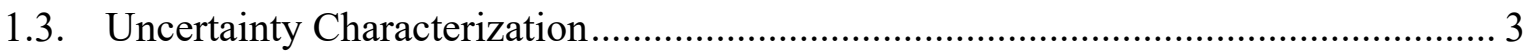

2. Mobile Manipulator Test and Measurement Setup and Procedure ............................... 3

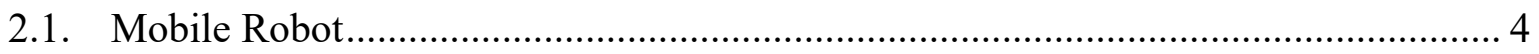

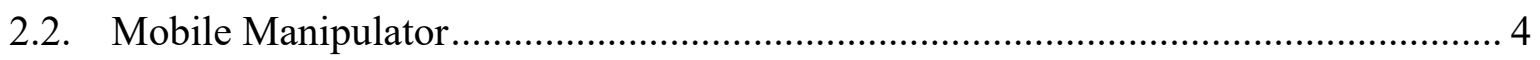

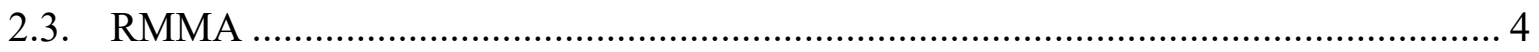

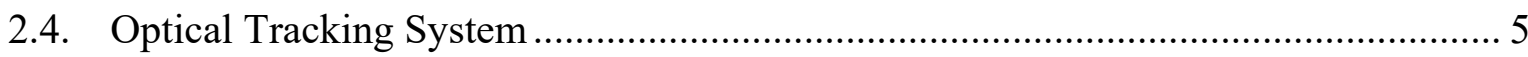

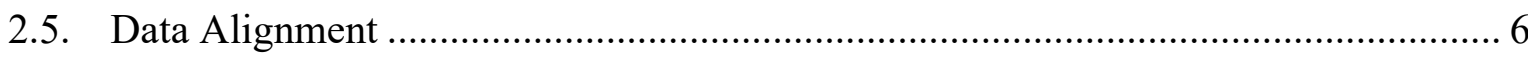

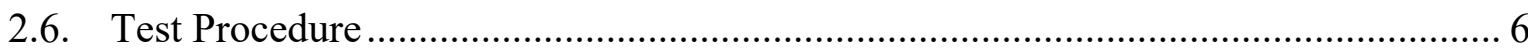

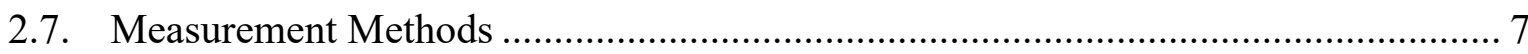

3. Coordinate System Registration of Mobile Manipulator to OTS .............................. 10

4. Mobile Manipulator Performance Test Method and Uncertainty Analysis ............ 13

4.1. Proposed Mobile Manipulator Test Method Using the RMMA ………………….... 13

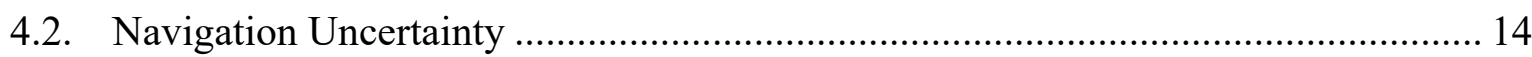

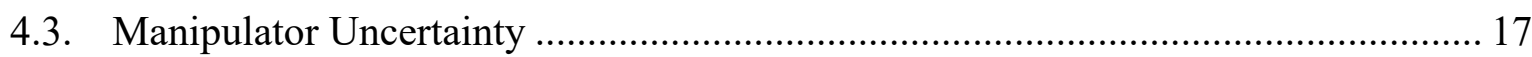

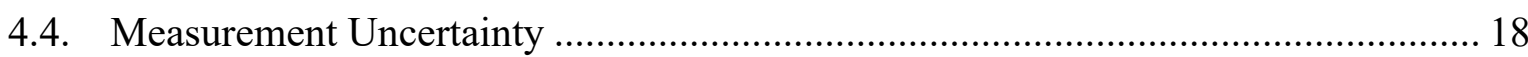

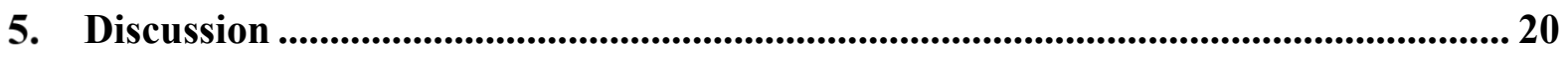

5.1. Measurement Method Uncertainty ……………….............................................. 21

5.2. Mobile Manipulator System Uncertainty …………………………………….... 22

6. Future Work ......................................................................................................................... 22

7. Conclusion ............................................................................................................................. 23

Acknowledgment................................................................................................................. 24

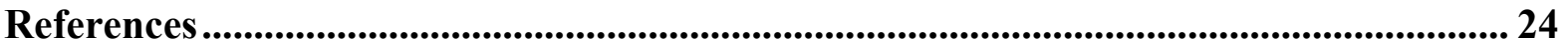




\section{List of Tables}

Table 1. Mobile base marker position stability ........................................................... 9

Table 2. Offset between computed positions of mobile base ........................................... 12

Table 3. Offset between the target positions from the manipulator and the OTS................ 13

Table 4. Mobile manipulator performance using the RMMA method ................................ 13

Table 5. Stability of the mobile base position. ................................................................... 15

Table 6. Measurement offset between a mobile base log and an OTS. ............................. 16

Table 7. Comparison of LR initial and final position offsets......................................... 17

Table 8. Comparison of AP manipulator position between manipulator log and OTS...... 19

Table 9. Mobile manipulator registration performance. ................................................. 20

\section{List of Figures}

Fig. 1. AGV mobile manipulator and advanced mobile manipulator................................. 2

Fig. 2. Top view of RMMA and mobile base showing positions of motion capture markers. 5

Fig. 3. Motion capture markers of mobile manipulator.................................................... 5

Fig. 4. Two planned path examples of the mobile base................................................. 7

Fig. 5. Motion capture tracking of manipulator's end-effector (EOAT) ........................... 8

Fig. 6. Mobile robot navigation and manipulator coordinate systems. .............................. 11

Fig. 7. Plot of the commanded positions, OTS measurements, and final analysis plot after coordinate system registration.

Fig. 8. Markers on the mobile base .............................................................................. 14

Fig. 9. OTS heading angle offset between mobile base and the RMMA........................... 15

Fig. 10. Plot of centroid positions for the mobile robot base, the manipulator (EOAT), and the RMMA targets as measured by the OTS.

Fig. 11. Plot of the centroid positions as logged by the mobile robot and manipulator registration, as well as the RMMA target locations in the EOAT coordinate frame. 


\section{Introduction}

Mobile robots have typically been utilized to transport materials [1][2] between workstations containing stationary robotic manipulators [3]. Alternatively, robotic manipulators can be moved between workstations [4] using an automatic guided vehicle $(A G V)$ or a mobile robot, which will be referred to in this paper as a mobile manipulator. The use of mobile manipulators can be advantageous in many situations. Mobile manipulators enable more agile manufacturing environments. For instance, it can result in cost savings when a single mobile manipulator replaces several stationary manipulators. Mobile manipulators offer increased flexibility and adaptability for rapidly evolving manufacturing task flows as the industry strives for continuous improvement in both the product quality and the manufacturing process efficiency. Mobile manipulators are useful in cases where efficiency gains are achieved by having the robots work around a large product rather than transporting the product through the factory floor to the stationary manipulators. Mobile manipulators also are more adaptable to the assembly of new product designs and manufacturing processes.

Mobile manipulators are appearing in manufacturing, healthcare, agriculture and other industries. The development of measurement methods and specific metrics to assess the safety, accuracy, and precision of mobile manipulators as systems including a mobile base with an onboard robot arm would provide means to ensure the systems can meet the constraints of the manufacturing processes [5]. Existing measurement methods have limitations and challenges in reconfigurability to assess a wide range of poses (positions and orientations) and a variety of mobile manipulator systems [6]. Current measurement techniques for individual components of the system, such as manipulators, include noncontact methods, such as laser interferometers, to contact-based measurement systems, such as artificial cues [6][7]. Research has primarily been focused on the stability of the manipulator and improving vehicle-manipulator control and coordination in field environments [8][9]. As technology advances from AGVs to mobile robots, additional challenges must be addressed. Mobile robots are designed to work in more variable environments without additional infrastructure to guide their route. However, this additional capability can contribute to greater positioning uncertainty and performance sensitivity to variations in the environment. Additionally, the lighter weight of the mobile base can contribute to greater instability of the manipulator payload. Methods to assess the uncertainty contributions of key components in mobile manipulators are needed.

\subsection{Prior Work}

In support of metrology for advanced mobile manipulators, the National Institute of Standards and Technology (NIST) Measurement Science for Manufacturing Robotics Program (MSMR) [15] has been researching performance measurement and test methods for mobile manipulators used in assembly applications. NIST has previously developed test and analysis methodologies to measure the manipulator-vehicle coordination, repeatability, and location accuracy critical to the mobile manipulator manufacturers and users of these relatively complex systems [10].

Research and development of novel test methods for mobile manipulators include the integration and comparison of: 1) the development of an optical tracking system (OTS) measurement method [16] as a means of establishing ground truth for mobile robot and 
manipulator positioning uncertainty, coordination between the manipulator and the vehicle and repeatability; 2) the development of a test method simulating flexible, reconfigurable manufacturing tasks that includes a relatively inexpensive reconfigurable mobile manipulator artifact (RMMA), as compared to an OTS, for use in measuring performance of mobile robots with an onboard manipulator [16]; and 3) the development of an analytical model for characterizing the measurement uncertainty of the RMMA to measure mobile manipulator performance using the OTS measurements as the ground truth [18]. Simulated and experimental test methods of applying RMMAs were developed for testing an AGV-based mobile manipulator's ability to locate the target, with measurement of position and alignment accuracy [6][17][26]. Computational methods for translating the target, manipulator and AGV coordinate frames to a world coordinate frame in order to assess performance were also developed and validated with errors less than $2 \mathrm{~mm}$ [22]. Finally, the AGV test method was improved by significantly reducing the search time with the integration of the bisect search method [26] of the AGV system (see Fig. 1(a)) using laser retroreflectors and simulated assembly points using reflective fiducials, enabling the manipulator equipped with a laser to automatically detect and $\log$ successful search events.
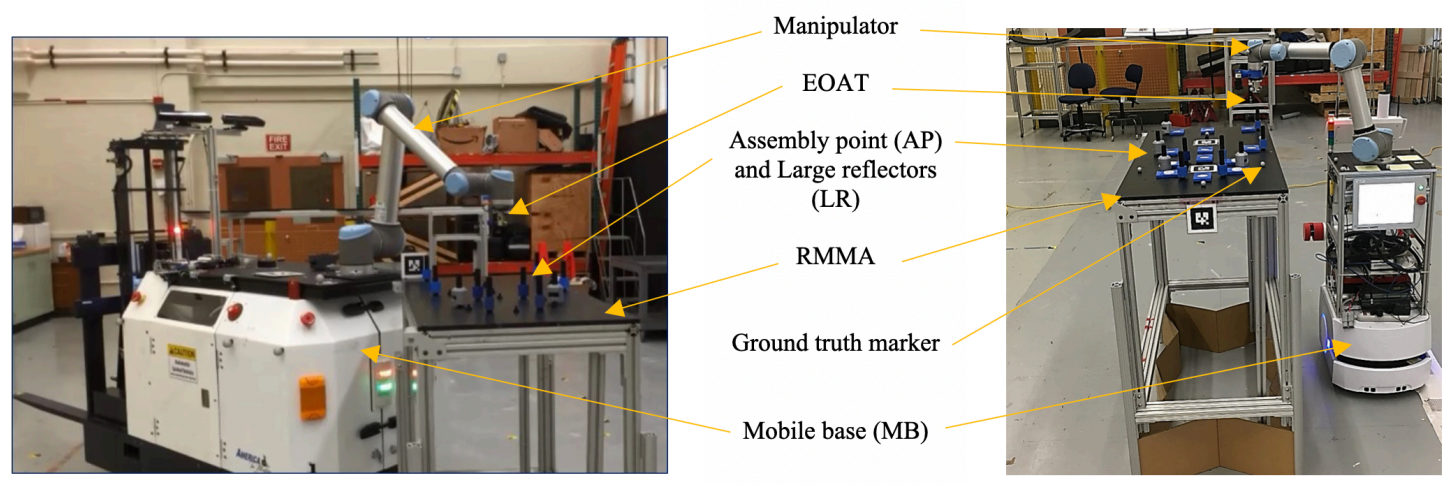

Fig. 1. (a) AGV mobile manipulator from previous research in 24. (b) Advanced mobile manipulator during the manipulator registration to the RMMA of this study.

\subsection{Research Contributions}

The purpose of this study was to demonstrate a measurement method using the RMMA to characterize advanced mobile manipulators (Fig. 1(b)). Seeking to further advance test and analysis methods based on Fig. 1(a)), this validation study used advanced mobile robots, which are designed to operate under more agile environments. The advanced mobile manipulator system included a mobile robot base with an onboard robotic manipulator having 6 degrees-of-freedom (DoF) operating in a simulated industrial environment using the RMMA to simulate reconfigurable assembly tasks. Improvements included the development of an advanced mobile manipulator and integration of precision time synchronization of the controllers with the OTS. The precision time synchronization capabilities were intended to support more accurate temporal alignment of the mobile base controller, the manipulator controller, and the OTS data sources. The experiment also required the theoretical determination of the two-dimensional (2D) position, on a plane, of the mobile manipulator end-of-arm-tool (EOAT) with respect to the experimental ground truth system reference. 


\subsection{Uncertainty Characterization}

The mobile manipulator performance test method analyzed the Euclidean distance of the target to the manipulator, the number of searches and the average and maximum number of search steps to locate the assembly points. The metrics supported an assessment of the accuracy and repeatability of the mobile manipulator to determine the target location. A comparison of two analysis methods were used to assess the aggregate and individual positioning and target registration repeatability of the mobile robot and the manipulator. The first test method utilized the RMMA with the respective controller logs to determine the mobile base position, the position stability, and the repeatability of the manipulator relative to the target assembly points. The second analysis method used the OTS to assess the uncertainty of the RMMA method by comparing the OTS position estimates of the mobile manipulator rigid bodies relative to the RMMA targets, deemed to be the ground truth, with the logged position estimates. The OTS simultaneously measured the RMMA target positions with an uncertainty of $0.02 \mathrm{~mm}$ and the mobile manipulator position after the target was located to estimate the mobile manipulator uncertainty for a simulated precision assembly task. By comparing the difference between the results from the RMMA to the results from the OTS, the study was able to benchmark the measurement uncertainty of the RMMA as a metrology tool for mobile manipulators. The study also demonstrated how the OTS can be used to assess the mobile robot base navigation uncertainty by comparing the results derived from the OTS data to the data from the mobile robot logs.

The paper first describes the experiment including the advanced mobile manipulator system, the RMMA, and the OTS. Following is a description of the data collected from each of the advanced mobile manipulator components. Next is a section describing the analysis methods to assess the positioning uncertainty of the advanced mobile manipulator followed by detailed results sections that tabulate and plot the analyzed data for comparison of logged robot data to ground truth to gauge the measurement bias and uncertainty between the logged robot data, the OTS, and the RMMA. The paper concludes with a discussion on the sources of measurement uncertainty, the potential sources of mobile manipulator uncertainty, and navigation uncertainty of advanced mobile manipulators in order to inform additional measurement sensors needed for future test method development, and for reducing the measurement error of future tests.

\section{Mobile Manipulator Test and Measurement Setup and Procedure}

In this section, we first describe the hardware and measurement equipment used in the experiment. Then we describe the non-contact experimental test procedure simulating a series of assembly tasks. The mobile manipulator test experiment to characterize the manipulator positioning uncertainty used a mobile manipulator performing a task to: 1) navigate to a goal adjacent to the RMMA, 2) stop at the goal to allow the manipulator to register to the RMMA, 3) detect assembly points on the RMMA, and 4) stow the manipulator, upon completion of the assembly operation, and index to the next goal. The target positions and search algorithms were programmed on the end effector controller, while the navigation and goal points were programmed on the mobile base controller. The position of the end effector was empirically measured using the OTS, which compared the position of the end effector's rigid body with the position of the RMMA target. The RMMA target positions were measured by replacing the targets with markers and taking a series of five static measurements over ten second intervals. The individual positions of the RMMA targets 
were compared to the position of the end effector after each successful search event. The comparison of measurements was used to evaluate the mobile manipulator's ability to detect the center of smaller targets, $2 \mathrm{~mm}$ diameter fiducials, for precision assembly tasks. The fiducials served as an approximation of the systems' assembly uncertainty and repeatability. For instance, we can track the number of end effector steps and successes for locating the 2 $\mathrm{mm}$ fiducial on the RMMA to estimate the repeatability within a $2 \mathrm{~mm}$ target uncertainty. The measurement experiment assessed the uncertainty of the position of the mobile robot system including the mobile robot base and the manipulator over ten trials using the OTS and the RMMA.

\subsection{Mobile Robot}

While AGVs typically use preprogrammed paths between navigational waypoints installed throughout the environment, advanced mobile robot navigation is based on internal intelligent route planning and positioning software with configurable parameters to specify an environment map. To develop this map, the mobile robot at NIST was manually driven using a joystick. The mobile robot detected objects in the experimental area using an internal laser scanning sensor that was approximately parallel with and approximately $10 \mathrm{~cm}$ above the floor. The map included the pose and stationary object features such as walls, partitions, fences, fixed manufacturing resources, and the RMMA structure.

Potential obstacles, such as the lower part of the RMMA, that could not be detected by the relatively low single-scan mobile robot sensors were manually added by the operator to the map. The experiment operator also denoted the charging station pose, the goals where the mobile robot should stop for performance measurement (simulating manufacturing assembly operations), and the driving constraints for guiding the mobile robot (e.g., only drive in one direction or along a defined path similar to an AGV). The mobile robot was shorter in height (by $118 \mathrm{~mm}$ ) with greater position uncertainty than the AGV used in prior research.

\subsection{Mobile Manipulator}

Fig. 1(b) shows the advanced mobile manipulator performing a simulated assembly task at the RMMA. The manipulator had a manufacturer-specified maximum reach of $850 \mathrm{~mm}$ and repeatability of $0.1 \mathrm{~mm}$ [24]. The onboard manipulator was controlled similarly to the one used in [2] and used a retro-reflective laser sensor and emitter to detect reflective targets without contact. The reflective targets were analogous to assembly points [17]. The laser sensor and emitter were mounted to the end-effector of the manipulator as shown in Fig. 1(b). The manipulator workspace was smaller than that of the previous manipulator. The advanced mobile manipulator required additional mobile base maneuvers to access a complete RMMA task simulation pattern.

\subsection{RMMA}

The RMMA was a $1.2 \mathrm{~m}$ x $0.6 \mathrm{~m}$ flat table structure constructed of anodized, machined aluminum. $42 \mathrm{~mm}$ diameter reflectors and $2 \mathrm{~mm}$ diameter cylindrical optical collimators, serving as target fiducials, were screwed into drilled holes on the RMMA. Each hole had a diameter uncertainty of $0.01 \mathrm{~mm}$. As illustrated in Fig. 2, the RMMA simulated a stationary task area with a $457 \mathrm{~mm}$ square pattern of four $2 \mathrm{~mm}$ diameter assembly point reflectors (each labeled Assembly Point, AP), which served as the targets. 


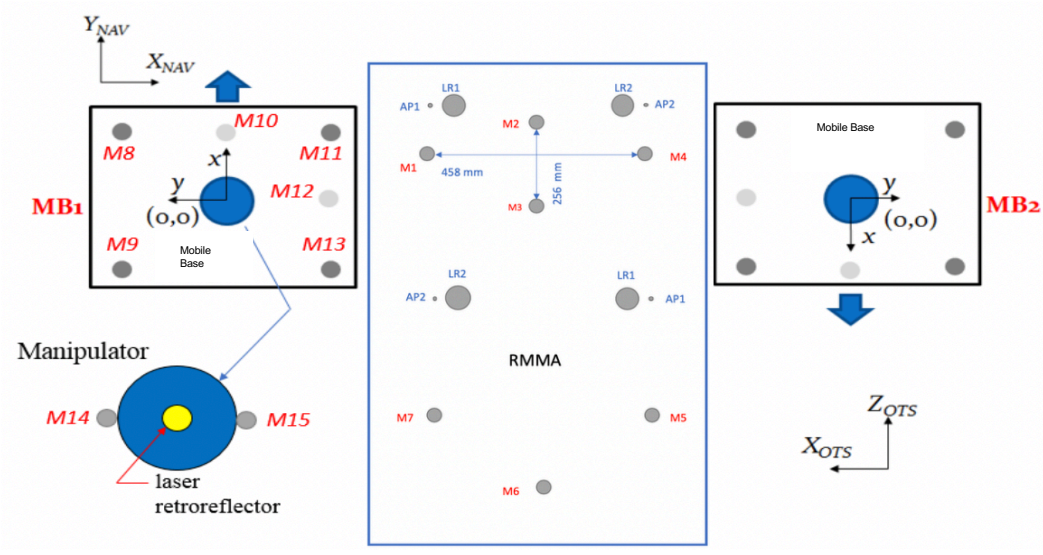

Fig. 2. Top view of RMMA and mobile base showing positions of the mobile base (MB), RMMA reflectors (AP and LR), and OTS markers (M). The 2D coordinate plane of the mobile base, the manipulator's laser retroreflector, and the RMMA are also shown.

\subsection{Optical Tracking System}

The OTS consisted of 20 synchronized cameras each with 4 mega-pixel resolution positioned throughout the $9 \mathrm{~m}$ wide $\times 22 \mathrm{~m}$ long $\times 7 \mathrm{~m}$ high laboratory. Out of the 20 cameras, eight had the most direct, relatively localized view of the RMMA. Additionally, one OTS camera was mounted to the ceiling directly above the RMMA. Prior to the experiment, the OTS was calibrated according to the manufacturer's procedures [23] using the Motive software version $1.10 .^{1}$ In order to establish the ground truth positions of the simulated target points, the eight AP and LR locations, the reflectors were removed, and an OTS marker was placed at each reflector location. The simulated task target positions were measured by the OTS within $0.02 \mathrm{~mm}$ uncertainty and used as ground truth to determine the manipulator offset from the target position. Fig. 3 shows the markers (illuminated by camera flash) placed on the RMMA and used for determining the ground truth positions of the assembly targets for the experiment.

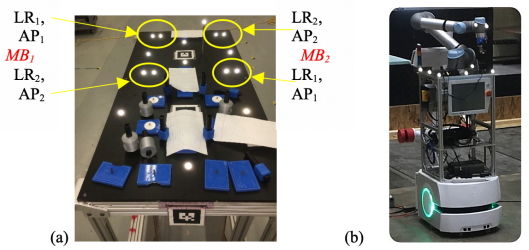

Fig. 3. (a) Markers (illuminated by camera flash) placed on the RMMA and used to determine ground truth target reflector 3D position measurements for the experiment. One additional marker (upper right) raised above the RMMA surface plane was not used in the experiment. (b) Mobile manipulator with markers (illuminated by camera flash) mounted on the base frame and manipulator.

\footnotetext{
${ }^{1}$ Certain commercial entities, equipment, or materials may be identified in this document in order to describe an experimental procedure or concept adequately. Such identification is not intended to imply recommendation or endorsement by the National Institute of Standards and Technology, nor is it intended to imply that the entities, materials, or equipment are necessarily the best available for the purpose.
} 


\subsection{Data Alignment}

The OTS computer used the W32Time service based on the Network Time Protocol (NTP) with the NIST local time servers providing the reference source. While the NTP clock synchronization can maintain time to better than $1 \mathrm{~ms}$ accuracy in local area networks under ideal conditions [19], this performance is not guaranteed by the time service application [20]. The OTS computer was also temporarily connected via Ethernet to the mobile robot and manipulator control laptops allowing local synchronization and timestamping of the logged data. The mobile robot and manipulator controllers communicated via the local wireless Ethernet network with the control computers, which logged the detection events. Delay between when the EOAT target detection event occurred and when the event was time-stamped at the controller was a potential source of data alignment uncertainty. Delay variability from wireless communication between both the mobile robot and its control computer and the manipulator's control computer may have existed, although it was assumed to be negligible. To ascertain the position of the EOAT upon target location, the controller was programmed to pause for a duration of $10 \mathrm{~s}$ to ensure sufficient data from the OTS and the mobile manipulator can be compared.

\subsection{Test Procedure}

Each simulated assembly task comprised of several sub-tasks. The first sub-task began with the mobile manipulator navigating to the first previously taught goal point (MB1) from a charging station. In the second sub-task, the mobile robot stopped at MB1, and the manipulator moved from a stowed pose through three interim poses to position an EOAT laser retroreflector at a previously taught registration point, LR1. In the third sub-task, the coordinate registration method used a coarse, spiral search method, to detect LR1. If LR1 was detected immediately, no coarse search method was required. Subsequently, a bisect search method of $0.5 \mathrm{~mm}$ steps was used to determine LR1's center [18].

The manipulator then moved to a second previously taught registration point (LR2) and repeated the search process for LR2. Two AP positions were also previously taught. Upon registration, the manipulator moved to AP1, where a $2 \mathrm{~mm}$ diameter reflector was located. The manipulator used a square spiral search pattern of $0.5 \mathrm{~mm}$ steps to detect AP1. Once detected, the manipulator moved to a second assembly point (AP2) and repeated the $2 \mathrm{~mm}$ diameter reflector detection process. To determine position repeatability, the manipulator searched again to detect AP1, followed by AP2, and then stowed, providing a total of 4 AP registration points per side.

The mobile robot proceeded to move to the opposite side of the RMMA to a second previously taught goal (MB2) and performed the second sub-task, a duplicate of the first subtask, to register and complete detection of the square pattern of assembly points. The entire task, including the two sub-tasks, was repeated for ten trials. The experiment had a total detection event set of 40 LR registration points ( 2 sides $\mathrm{x} 2 \mathrm{LR}$ registration points per side $\mathrm{x} 10$ repetitions per side) and 80 AP registration points $(2$ sides $x 4$ AP registration points per side $\mathrm{x} 10$ repetitions per side). Fig. 4 (a) and (b), respectively, show the two planned paths of the mobile base ready to move from MB1 to MB2 and from MB2 to MB1. 


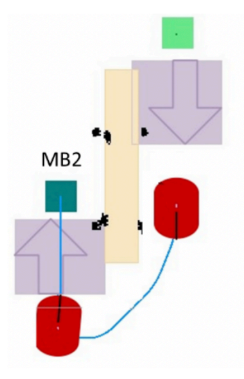

(a)

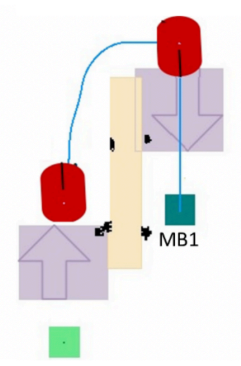

(b)

Fig. 4. Two planned path examples of the mobile base ready to move from (a) MB1 to MB2 and (b) from MB2 to MB1. Legend: red oval represents the vehicle, violet arrows denote the vehicle's direction, black denotes the RMMA structural posts as detected by the vehicle, green squares are interim stop points in-line with the nearest goal (MB1 or MB2), blue lines are planned paths by the mobile base, beige rectangles denote the region where the mobile base was restricted from access.

\subsection{Measurement Methods}

Two methods were used to measure the mobile manipulator task performance. Method 1 uses the RMMA, the mobile base, and the manipulator controller logs of the two registration points on the RMMA to determine the initial-to-final mobile manipulator EOAT offsets at the two LRs as the mobile manipulator stopped to access the RMMA for the registration and assembly process. This method assesses the distance of the advanced mobile manipulator's EOAT between the start and final position using the RMMA. The distance obtained from the controller logs were compared with the distance from the OTS measurement to determine the overall error and uncertainty of the RMMA method. The registration coordinates were used to detect two assembly points twice (i.e., four detections). Each detection event of the registration and assembly points were recorded within the manipulator log files.

Three advanced mobile manipulator performance metrics were characterized as part of the test procedure used to assess the RMMA. The first metric was the docking performance of a mobile robot to the MBs. To characterize a mobile robot docking performance to the MBs, an analysis method was proposed to determine the error of the mobile robot base position with respect to the commanded position (goal) and the actual position. The second metric was the registration performance of the mobile manipulator to the RMMA. For the test procedure, a proposed metric was to compute the distance between the initial manipulator registration position to the LR on the RMMA. The third metric was assessing a manipulator's performance by computing a manipulator's distance to the programmed assembly point on the RMMA. The manipulator's distance from the target can be used to determine whether the registration step, after bisecting each LR, was sufficient to allow the manipulator to immediately detect each AP as well as the magnitude of the offset from the target.

The second method, using the OTS, assessed the error and the uncertainty of the RMMA method. The OTS simultaneously measured the mobile base and the EOAT relative to the RMMA target ground truth positions. To measure the mobile base, the EOAT, and the RMMA target positions and orientations at each programmed or detection event using the 
OTS, the manipulator paused $10 \mathrm{~s}$ at 1) the initial search for each LR, 2) the final LR center point after the bisect search, and 3) the final AP point after LR bisect, or if needed, after a fine search to detect the AP.

Data analysis for the OTS method consisted of comparing the same qualifications as in the RMMA method. Critical to the analysis of this method was the data selection method, ensuring the position data were based on the time when the EOAT detected the target and when the mobile manipulator was stationary, to minimize the measurement uncertainty of the EOAT position. The EOAT was programmed to pause for $10 \mathrm{~s}$ at the following target points: LR1 Initial, LR2 Initial, LR1 Final, LR2 Final, AP1 (first), AP2 (first), AP1 (second), AP2 (second). The standard deviations of the $x$ and $y$ coordinates of the EOAT markers, specifically markers M14 and M15 as shown in Fig. 5, were used to assess the stability of the manipulator and to determine the window of data to be used to compare the manipulator and target distances.

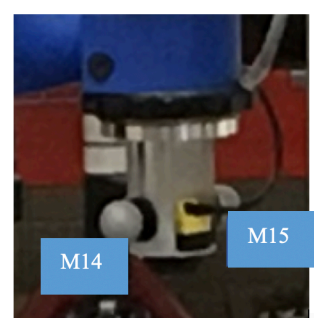

(a)

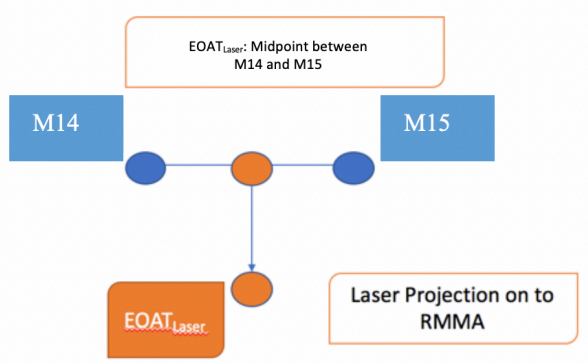

(b)

Fig. 5. (a) EOAT markers M14 and M15. (b) Midpoint (EOAT laser $_{\text {) }}$ used to estimate the target position. 
The OTS data selection used an iterative approach to determine when the EOAT was in the paused position. The algorithm was based on two key assumptions, namely (1) during the pause, the EOAT had minimal jitter and (2) there may be some settling time for the EOAT to fully stabilize at the beginning of the pause. Therefore, after $1 \mathrm{~s}$ to $2 \mathrm{~s}$ in the paused position, the uncertainty of the marker positions on the EOAT should be the combined uncertainty from jitter in the mobile robot and the static OTS measurement uncertainty, assuming there were minimal extraneous motions of the mobile robot or the manipulator. The standard deviations, $\sigma_{\mathrm{EOAT}_{x}}$ and $\sigma_{\mathrm{EOAT}_{y}}$, were taken during a $5 \mathrm{~s}$ sliding window of data or 600 contiguous frames at 120 frames per second (fps) OTS data acquisition rate. The $\sigma$ threshold values were iteratively increased starting at $0.1 \mathrm{~mm}, 0.5 \mathrm{~mm}, 1 \mathrm{~mm}$, and up to $2 \mathrm{~mm}$ until all eight points of interest were found for all 20 trials. The thresholds were based upon the stability in the mobile robot markers (see Table 1), the uncertainty of the OTS based on the stationary ground truth data (see Table 7), and the stability in the manipulator. Previous experiments demonstrated that the OTS used has a static measurement uncertainty of $0.02 \mathrm{~mm}$ and $0.05^{\circ}$ and a dynamic measurement uncertainty of $0.26 \mathrm{~mm}$ and $0.20^{\circ}$ [21].

Table 1. Mobile base marker position stability within a trial for MB1 and MB2 (in $\mathrm{mm}$ ).

\begin{tabular}{|l|l|l|l|}
\hline $\begin{array}{l}\text { Mobile Base } \\
\text { Markers }\end{array}$ & Mean $_{x}[$ stdev] & Mean $_{y}[$ stdev] & Mean $_{z}[$ stdev] \\
\hline Marker 10 (MB1) & $-132.09[0.17]$ & $5583.24[0.13]$ & $1151.01[0.01]$ \\
\hline Marker 12 (MB1) & $-335.61[0.18]$ & $5403.16[0.12]$ & $1154.95[0.01]$ \\
\hline Marker 10 (MB2) & $-1731.31[0.15]$ & $5303.12[0.24]$ & $1177.49[0.29]$ \\
\hline Marker 12 (MB2) & $-1525.65[0.14]$ & $5480.79[0.27]$ & $1177.83[0.01]$ \\
\hline
\end{tabular}

We analyzed the stability of the mobile robot and determined the stability thresholds as indicated in Table 1, when the mobile robot was stationary. If the EOAT marker coordinates for M14 and M15 were determined by the thresholding algorithm to be within the paused time frame, the algorithm proceeded to compute the midpoint to provide the paused position as shown in (1), as the 2D manipulator target position.

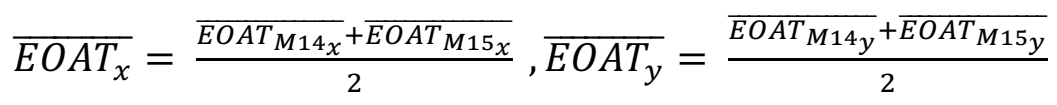

The test operator also manually recorded the timestamp of when the first two initial bisects occurred and when the APs were registered, as a means to verify the approximate time alignment to the OTS data. The test operator recorded the time from the OTS computer to a $1 \mathrm{~s}$ resolution. The timestamps were used to verify that the points detected were within the $5 \mathrm{~s}$ sliding window of the data logged by the OTS. The algorithm allowed for error as large as $1 \mathrm{~s}$ due to the operating system clock display resolution.

Some of the challenges encountered in the experiment included the large threshold value and the time alignment that creates additional uncertainty of the exact position of the EOAT. The instability in the base can further propagate uncertainty of the manipulator target position. The time alignment issue can be improved with automated, timestamped data 
acquisition of the registration events onboard the advanced mobile manipulator to ensure temporal alignment of the full set of experimental data acquisition systems.

\section{Coordinate System Registration of Mobile Manipulator to OTS}

The logged data from the mobile base and manipulator control programs were analyzed with respect to the mobile base coordinate system specified when the mobile robot environment map was developed. The OTS coordinate system was established during calibration immediately prior to the experiment and was independent of the mobile base coordinate system. The mobile robot and manipulator logged data included a single timestamped position coordinate of when each of the various detection events occurred, including MB, initial and final LRs, and APs. The OTS logged data included a subset of rigid body position and orientation data of each mobile manipulator rigid body over the paused interval. Using the logged pose data from the mobile robot and the manipulator control programs and from the OTS ground truth measurement system, the data sets were then registered to a single coordinate system so that the logged data from the controller could be directly compared to the ground truth data from the OTS.

Data from the mobile manipulator consisted of position and orientation data representing the pose of the mobile base with respect to a world (Nav) coordinate frame, as well as the 2D position of the EOAT with respect to the manipulator base. The OTS measurements provided the ground truth and were based on markers placed on the mobile base, the EOAT, as well as on the RMMA to compute each component's position and orientation information.

First, the coordinate frames of the mobile base and of the EOAT were transformed to the OTS coordinate system. The mobile robot and manipulator each logged its pose data based on its own reference coordinate system. The mobile robot's coordinate system covered the RMMA location, but the manipulator can only cover its work volume. Therefore, to describe RMMA target positions using the mobile robot and the manipulator logged data, it was necessary to combine the coordinate systems of the mobile robot and the manipulator into one system.

The manipulator's positive $x$-axis was in the forward direction of the mobile base and the manipulator's positive $y$-axis is $90^{\circ}$ counterclockwise from its positive $x$-axis. RMMA access for MB1 and MB2 were different in the mobile robot's coordinate system, and the manipulator EOAT log was converted to the mobile robot's coordinate system. Meanwhile, a misalignment occurred when the mobile robot stopped at MB1 or MB2 relative to the RMMA (see Fig.). The manipulator logged the EOAT pose data under this error condition, therefore it was necessary to rotate the EOAT pose to describe it in the mobile robot's coordinate system. As shown in Fig., the manipulator EOAT pose was converted using (2) and (3) for MB1 and MB2, respectively.

$$
\begin{gathered}
\operatorname{EOAT}_{M B 1}= \\
\left(L_{x}-M_{y} * \cos (\theta)-M_{x} * \sin (\theta), L_{y}-M_{x} * \cos (\theta)+M_{y} * \sin (\theta)\right) \\
\operatorname{EOAT}_{M B 2}= \\
\left(L_{x}+M_{y} * \cos (\theta)+M_{x} * \sin (\theta), L_{y}-M_{x} * \cos (\theta)+M_{y} * \sin (\theta)\right)
\end{gathered}
$$




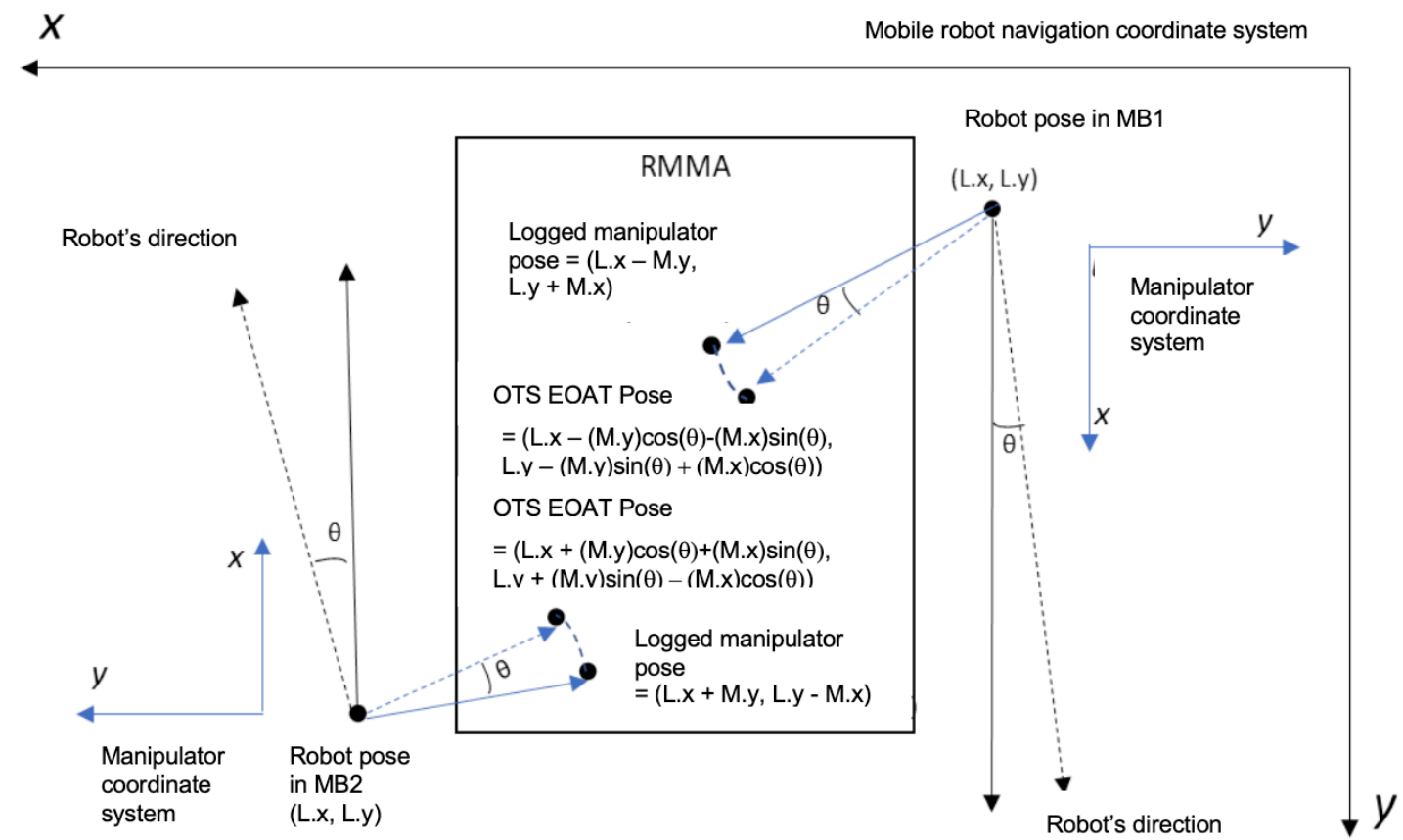

Fig. 6. Mobile robot navigation and manipulator coordinate systems.

Then, data from the mobile manipulator's coordinate system was transformed into the OTS coordinate system in order to compare the mobile manipulator data to assess the distance offset between the mobile robot's commanded position, its final logged position, and its actual position as measured by the OTS. This was accomplished by solving the registration problem as shown in (4) [25]:

$$
\min _{H}\|H O-N\|^{2}
$$

where $O=\left[O_{1}, O_{2}, \ldots, O_{n}\right]$ and $N=\left[N_{1}, N_{2}, \ldots, N_{n}\right]$. Here $O_{i}$ and $N_{i}$, for $i=1,2, \ldots n$, are homogeneous transformation matrices representing the $2 \mathrm{D}$ pose of the mobile manipulator with respect to the OTS and Nav coordinate system, respectively.

The registration between the OTS and Nav coordinate systems for the experimental setup was found to be:

$$
H=\left[\begin{array}{ccc}
0.07 & -1.00 & 5608.11 \\
1.00 & 0.07 & -6803.14 \\
0 & 0 & 1
\end{array}\right]
$$

or an orientation change of $-86.18^{\circ}$, and a translational offset of $5608.11 \mathrm{~mm}$ in the $x$ direction, and $-6803.14 \mathrm{~mm}$ in the $y$-direction. The commanded positions (MB1, MB2) were then compared with the data obtained from the Nav system and the registered OTS system (see Fig. (a)). The mean and standard deviation of the difference is shown in Table 2. 
Table 2. Mean and [standard deviation] of the distance between the commanded and measured positions of the mobile base. The navigation system position is compared with the registered OTS position ( $\mathrm{mm}$ ).

\begin{tabular}{|l|l|}
\hline Nav/OTS & Mobile Base Distance \\
\hline Nav MB1 & $25.03[10.42]$ \\
\hline Nav MB2 & $22.34[10.31]$ \\
\hline OTS MB1 & $32.34[15.09]$ \\
\hline OTS MB2 & $40.02[21.22]$ \\
\hline
\end{tabular}

The Nav data and the commanded positions were better aligned than those computed from the OTS system. The results signified that the mobile base assumed it was reaching the commanded positions more accurately than it actually was.

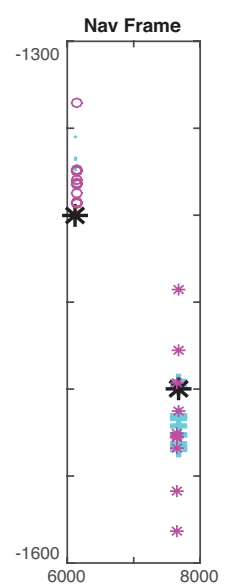

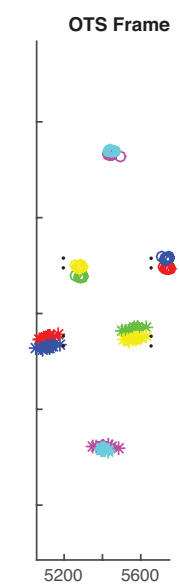

(a)

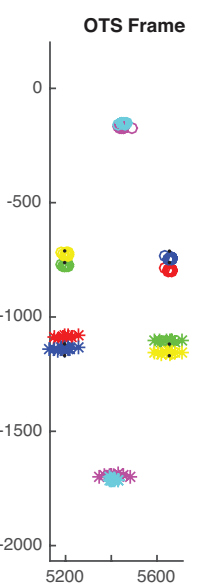

(b)

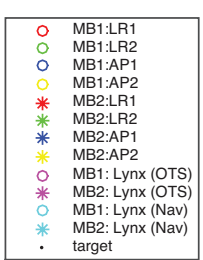

(c)

Fig. 7. (a) Plot of the commanded positions (black asterisks) for MB1 and MB2 compared to the data obtained from the Nav system and the registered OTS system. (b) Plot of the mobile base location relative to the OTS coordinate frame using registration $H$. (c) Final analysis plot after incorporating the registration.

The manipulator was then assumed to be centered on the mobile base coordinate system, and used to compute the positions of the LR1, LR2, AP1, and AP2 targets from the two commanded positions in the Nav coordinate frame. Using the registration, $H$, the manipulator data was registered to the OTS coordinate frame to compare with the ground truth system (see Fig. (b)). There appeared to be an offset between the ground truth OTS targets and the computed LR1, LR2, AP1, and AP2 targets. As a result, an additional registration was computed to determine the offset between the mobile base coordinate system and manipulator's base coordinate system, which consisted of a rotation offset of $-7.82^{\circ}$ and a translational offset of $0.35 \mathrm{~mm}$ in the $x$-direction and $2.10 \mathrm{~mm}$ offset in the $y$-direction. This offset was incorporated to determine the final analysis (see Fig. (c)). The mean and standard deviation of the difference from the final computed LR1, LR2, AP1, and AP2 targets from 
the Nav coordinate system with the OTS targets from the commanded MB1 and MB2 positions are shown in Table 3.

Table 3. Mean and [standard deviation] of the distance between the final computed LRs and APs from the Nav coordinate system and the OTS targets (mm).

\begin{tabular}{|l|l|l|}
\hline Goal & LR/AP & Manipulator Distance \\
\hline MB1 & $L R 1$ & $33.70[3.04]$ \\
\hline & $L R 2$ & $16.31[3.35]$ \\
\hline & $A P 1$ & $32.94[2.94]$ \\
\hline & $A P 2$ & $17.06[2.95]$ \\
\hline MB2 & $L R 1$ & $40.48[13.00]$ \\
\hline & $L R 2$ & $32.69[19.57]$ \\
\hline & $A P 1$ & $44.24[15.40]$ \\
\hline & $A P 2$ & $32.98[20.01]$ \\
\hline
\end{tabular}

\section{Mobile Manipulator Performance Test Method and Uncertainty Analysis}

Experimental results were determined upon registration of the two coordinate systems from the mobile robot's navigation map and from the OTS coordinate system. The following sections describe the mobile manipulator performance measured by RMMA method. This section describes in greater detail the uncertainties of the logged mobile robot position and the logged EOAT position as compared between OTS and RMMA methods.

\section{1. $\quad$ Proposed Mobile Manipulator Test Method Using the RMMA}

The objective of the simulated assembly task was to find the APs. The mobile manipulator system succeeded to complete the task for all 40 trials. Using the RMMA setup, mobile robot logs and EOAT logs, the observed mobile manipulator positioning offset are shown in Table 4. Errors of $x=4.65 \mathrm{~mm}, \mathrm{y}=22.60 \mathrm{~mm}$, and $1.00^{\circ}$ heading in docking performance were logged by the mobile robot. The errors were propagated to the initial EOAT pose errors of $x=21.46 \mathrm{~mm}$ and $\mathrm{y}=16.21 \mathrm{~mm}$. During 2 trials, the EOAT did not detect the APs immediately after the registration, due to registration errors of $\mathrm{x}=0.20 \mathrm{~mm}$ and $\mathrm{y}=0.12 \mathrm{~mm}$. There were 6 cases when EOAT failed to find the known APs immediately (second search), therefore requiring an additional spiral search. This was due to the observed manipulator positioning errors of $\mathrm{x}=0.07$ and $\mathrm{y}=0.03 \mathrm{~mm}$.

Through the test and analysis, it was verified that the RMMA method can measure the performance of an advanced mobile manipulator for locating $2 \mathrm{~mm}$ assembly targets. The next sections describe the uncertainty of each system component using the ground truth motion capture measurement system. Analysis was conducted separately for MB1 and MB2 to further understand the potential sources of navigation, manipulator, and measurement uncertainty. 
Table 4. Mobile manipulator metrics and observed performance using the RMMA method.

\begin{tabular}{|c|c|c|c|}
\hline Tasks & Metric & How to measure & Performance by RMMA \\
\hline Docking to MBs & Docking offset & Commanded pose vs docked pose & $\begin{array}{c}\text { mean }(\mathrm{std}) \\
\mathrm{x}: 4.65(2.15) \mathrm{mm} \\
\mathrm{y}: 22.60(11.35) \mathrm{mm} \\
\text { Theta: } 1.00(0.52)^{\circ}\end{array}$ \\
\hline Finding LRs & Registration offset & $\begin{array}{c}\text { Distance between initial and final } \\
\text { EOAT pose to find LRs }\end{array}$ & $\begin{array}{c}\text { \# of success search: } 40 / 40 \\
\text { mean(std) } \\
\text { x: } 21.46(6.32) \mathrm{mm} \\
\mathrm{y}: 16.21(4.00) \mathrm{mm}\end{array}$ \\
\hline Finding APs (first) & Registration offset & $\begin{array}{c}\text { Distance between initial and final } \\
\text { EOAT pose to find APs }\end{array}$ & $\begin{array}{c}\text { \# of success search: } 40 / 40 \\
\text { mean(std) } \\
\text { x: } 0.20(0.91) \mathrm{mm} \\
\mathrm{y}: 0.12(0.55) \mathrm{mm}\end{array}$ \\
\hline $\begin{array}{l}\text { Finding APs } \\
\text { (second) }\end{array}$ & Positioning precision & $\begin{array}{c}\text { Distance between initial and final } \\
\text { EOAT pose to find APs }\end{array}$ & $\begin{array}{c}\text { \# of success search: } 40 / 40 \\
\text { mean(std) } \\
\text { x: } 0.07(0.48) \mathrm{mm} \\
\mathrm{y}: 0.03(0.13) \mathrm{mm}\end{array}$ \\
\hline
\end{tabular}

\subsection{Navigation Uncertainty}

For both the mobile robot position and mobile robot orientation offset, 10,000 frames of data were used to ensure the mobile manipulator was stationary. The method to compute the mobile robot's pose was based upon the diagonals formed by the four markers, M8, M9, M13, and M11, mounted on the top rectangular shelf of the mobile robot structure, as shown in Fig. .

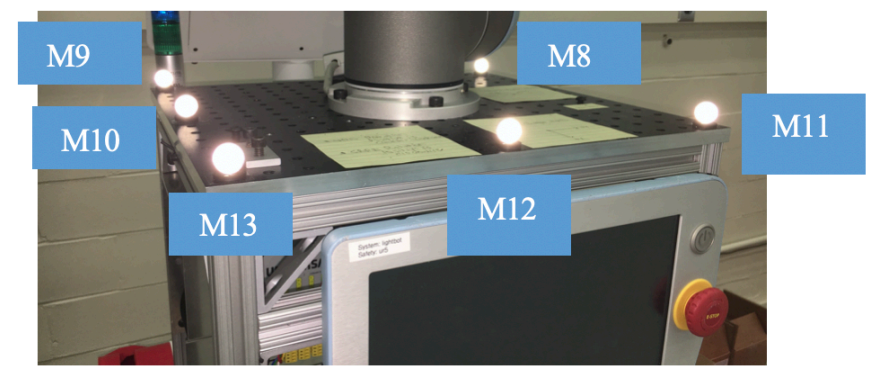

Fig. 8. Markers on the mobile base.

The midpoint of the diagonal lines was computed between markers M8 and M13 and between markers M9 and M11. The two midpoints were averaged to determine the mobile base pose centroid. A single trial showed a difference of about $1 \mathrm{~mm}$ to $2 \mathrm{~mm}$ in the two diagonal midpoints (see Table 5), signifying the $x y$ plane of the EOAT coordinate system may not be completely aligned with the plane of the mobile base coordinate system. 
Table 5. Stability of the mobile base position, expressed as mean [standard deviation] (in $\mathrm{mm}$ ) in a single trial with base held for 10000 frames at $120 \mathrm{fps}$.

\begin{tabular}{|c|l|l|}
\hline $\begin{array}{l}\text { Mobile Base } \\
\text { Position }\end{array}$ & $\begin{array}{l}\text { Mobile Base } \\
\text { Centroid 1 }\end{array}$ & $\begin{array}{l}\text { Mobile Base } \\
\text { Centroid 2 }\end{array}$ \\
\hline $\mathbf{x}$ & $-132.88[0.10]$ & $-131.89[0.10]$ \\
\hline $\mathbf{y}$ & $5404.79[0.13]$ & $5406.22[0.13]$ \\
\hline $\mathbf{z}$ & $1149.77[0.05]$ & $1149.62[0.05]$ \\
\hline
\end{tabular}

The mobile robot pose angle offset from the RMMA, $\theta_{\text {mobile_base }}$, was computed using (5). The vectors were formed from the mobile robot's OTS markers M11 and M13, and the RMMA OTS markers M2 and M3, as shown in Fig. and Fig. .

$$
\theta_{\text {mobile_base }}=\cos ^{-1}\left(\frac{\left(V_{13 \_11} \cdot V_{4 \_2}\right)}{\widehat{V}_{13 \_11} \times \widehat{V}_{4 \_} 2}\right) \times \frac{180}{\pi}
$$

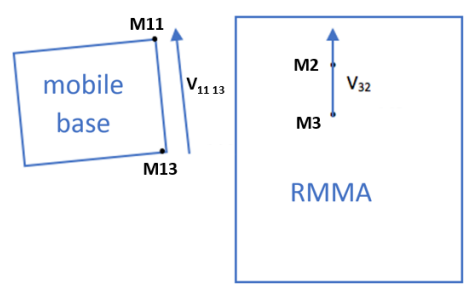

Fig. 9. Vectors used for the OTS heading angle offset between mobile base and the RMMA.

Fig. 10. Plot of centroid positions for the mobile robot base, the manipulator (EOAT), and the RMMA targets as measured by the OTS. shows a plot of centroid positions as measured by the OTS for the mobile base and the EOAT as well as the RMMA target locations for the experiment described in Section II. As expected, the final EOAT positions coincided with the mobile base positions where the manipulator extended to the expected RMMA AP position. 


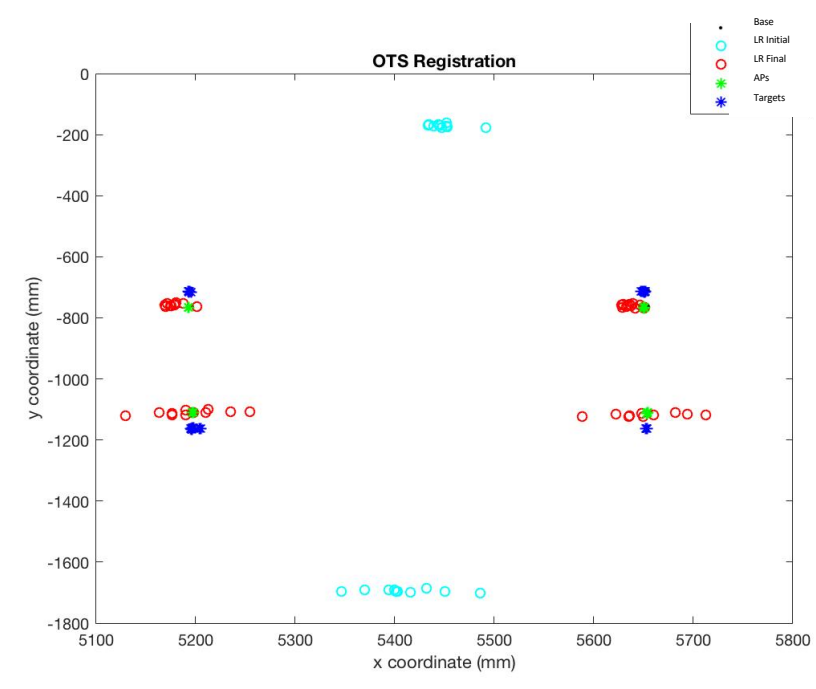

Fig. 10. Plot of centroid positions for the mobile robot base, the manipulator (EOAT), and the RMMA targets as measured by the OTS.

Table 6 shows the mean and the standard deviation of the offset between the logged Nav positions and the OTS measured positions. Column A shows the commanded mobile robot positions and column B shows where the mobile robot logged its position. The range [and standard deviation] of mobile robot position offsets logged by the mobile robot controller relative to the commanded poses are shown in Table 6 as column D. Additionally, column C of Table 6 shows the location where the OTS measured the actual position of the mobile robot in the OTS coordinate system. Column E compares the OTS measured mobile base position to the mobile robot logged pose, which showed a relatively large bias between the final mobile robot logged position relative to its position estimated by the OTS.

Table 6. Mean bias and [standard deviation] of the mobile base navigation system and the registered OTS with the commanded poses $(\mathrm{mm})$.

\begin{tabular}{|c|c|c|c|c|c|c|}
\hline \multirow{5}{*}{} & Units & $\begin{array}{c}\text { (A) } \\
\text { Commanded } \\
\text { mobile base } \\
\text { pose }\end{array}$ & $\begin{array}{c}\text { (B) Logged } \\
\text { mobile base } \\
\text { pose }\end{array}$ & $\begin{array}{c}\text { (C) OTS - } \\
\text { mobile base } \\
\text { pose (referenced } \\
\text { to mobile base } \\
\text { Nav) }\end{array}$ & $\begin{array}{c}\text { (D=B-A) } \\
\text { Commanded } \\
\text { vs. logged }\end{array}$ & (E=B-C) OTS vs. logged \\
\hline \multirow{4}{*}{ MB1 } & $x(\mathrm{~mm})$ & 6120 & $6124.4[2.3]$ & $6160.1[5.6]$ & $4.4[2.3]$ & $-35.7[5.4]$ \\
\cline { 2 - 7 } & $y(\mathrm{~mm})$ & -1400 & $-1375.8[11.3]$ & $-1365.8[16.3]$ & $24.2[11.3]$ & $10.0[10.0]$ \\
\cline { 2 - 7 } & $\begin{array}{c}\text { Heading } \\
\left({ }^{\circ}\right)\end{array}$ & 90.0 & $90.6[0.5]$ & $90.8[0.5]$ & $0.6[0.5]$ & $-0.2[1.0]$ \\
\hline \multirow{4}{*}{ MB2 } & $x(\mathrm{~mm})$ & 7675 & $7676.7[5.3]$ & $7681.0[5.6]$ & $1.7[5.3]$ & $-4.3[5.1]$ \\
\cline { 2 - 7 } & $y(\mathrm{~mm})$ & -1500 & $-1520.0[13.6]$ & $-1512.0[39.5]$ & $-20[13.6]$ & $8.0[33.4]$ \\
\cline { 2 - 7 } & $\begin{array}{c}\text { Heading } \\
\left({ }^{\circ}\right)\end{array}$ & -90 & $-88.9[0.9]$ & $-90.7[0.6]$ & $1.1[0.9]$ & $-1.7[0.4]$ \\
\hline
\end{tabular}

The mean mobile robot offsets from commanded poses, considering the logged data versus the commanded positions (i.e., Table 6, column D) for MB1 and MB2, were $x=4.4$ $\mathrm{mm}, y=24.2 \mathrm{~mm}, \theta=1.1^{\circ}$ with respective standard deviations of $x=2.3 \mathrm{~mm}, y=11.3 \mathrm{~mm}$, and $\theta=0.9^{\circ}$. The uncertainty computed from the difference between the logged and OTS data were, respectively, $x=5.7 \mathrm{~mm}, y=16.4 \mathrm{~mm}$, and $\theta=0.6^{\circ}$. The maximum position 
offset of the mobile robot, as indicated by the OTS, was approximately $40 \mathrm{~mm}$ from commanded $y$ position versus the logged error of $13.6 \mathrm{~mm}$. Comparing the logged (robot) and computed (OTS) mobile base positions in $x$ and $y$, the maximum position offset observed in the experiment on each side, MB1 and MB2, shown in Table 6, column E, were $8.0 \mathrm{~mm}$ and $35.7 \mathrm{~mm}$, respectively.

\subsection{Manipulator Uncertainty}

In addition to the mobile base position uncertainty, which can propagate to the EOAT, the EOAT also has its own positioning uncertainty. Since the errors from the mobile robot were propagated through registration, the uncertainty was derived from the standard deviation of the difference between the two independent, random final and initial LR positions. Table 7 compares the mean LR initial and final offsets from the manipulator and OTS logs. The large standard deviation of approximately $21 \mathrm{~mm}$ are potentially due (1) OTS measurement uncertainty, caused by the OTS marker placement and (2) the variability and uncertainty propagation of the mobile robot and manipulator positions between trials.

Based on the RMMA height assessment between markers closer to MB1 and markers closer to MB2, there appears to be a slight slope of the floor that the RMMA rests on. The slope may contribute to greater variability in the manipulator's target positions on the MB2 side. Fig. and Fig. 6 illustrate the observed differences between the mobile robot logged data and the ground truth RMMA target position data (OTS). In Fig. 6, there was a shift in target locations in the EOAT coordinate frame, where the targets were actually in a $457.2 \mathrm{~mm}$ by $457.2 \mathrm{~mm}$ square pattern as shown from the OTS data in Fig. . The figures were scaled down in the y-axis to fully capture the mobile manipulator's position.

Table 7. Comparison of LR initial and final position offsets.

\begin{tabular}{|l|l|l|l|}
\hline \multirow{2}{*}{} & \multicolumn{3}{|l|}{$\begin{array}{l}\text { LRfinal }- \text { LR initial } x, y \text { offsets } \\
\text { mean [standard deviation] (in mm) }\end{array}$} \\
\cline { 2 - 4 } & & MB1 & $M B 2$ \\
\hline \multirow{3}{*}{ Manipulator Log } & LR1 & $14.9[5.9], 4.9[3.4]$ & $27.6[21.0], 5.8[[3.8]$ \\
\cline { 2 - 4 } & LR2 & $15.6[5.9], 7.5[4.5]$ & $27.6[21.4], 7.0[4.3]$ \\
\cline { 2 - 4 } & Overall & $15.3[5.8], 6.2[4.1]$ & $27.6[20.6], 6.4[4.0]$ \\
\hline \multirow{3}{*}{ OTS Log } & LR1 & $14.7[6.2], 6.7[4.1]$ & $28.6[21.0], 6.0[4.1]$ \\
\cline { 2 - 4 } & LR2 & $15.7[6.1], 7.6[4.6]$ & $27.7[21.6], 7.0[4.0]$ \\
\cline { 2 - 4 } & Overall & $15.2[6.0], 7.2[4.3]$ & $28.1[20.7], 6.5[4.0]$ \\
\hline
\end{tabular}

The performance of the mobile manipulator can be equated to how close the EOAT was positioned by the manipulator's expected final locations as compared to its initial position. This implies that the manipulator must: 1) know the current EOAT position, 2) know the expected final position, and 3) move to the expected final position with the exact distance and direction. 


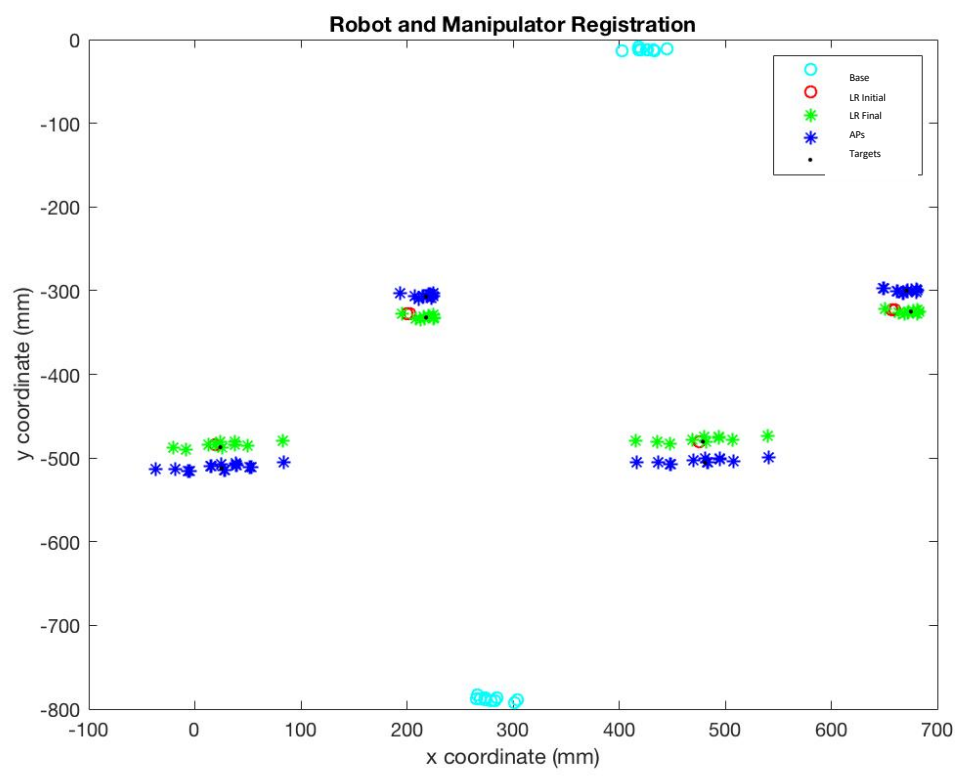

Fig. 6. Plot of the centroid positions as logged by the mobile robot and manipulator registration, as well as the RMMA target locations in the EOAT coordinate frame.

The uncertainty of the manipulator EOAT, comprised of the bias and standard deviation, was calculated by analyzing logged data and comparing it to OTS ground truth data. The EOAT pose uncertainty included the mobile robot uncertainty, since manipulator base pose errors occurred each time the mobile robot stopped. Thus, even if the manipulator moved the EOAT with the same pose command on two different trials, the actual EOAT pose differed because of the error propagated from the position of the mobile base. It was possible the environment, including variations in the floor surface levelness and floor surface friction of the test area, could have impacted the repeatability of the mobile manipulator's position.

For instance, in the 3D OTS position measurements of the RMMA LR and AP locations, the heights of the targets differed as much as $9 \mathrm{~mm}$ between the two MB sides. To analyze and evaluate the uncertainty of the manipulator EOAT, it must be isolated from the mobile robot uncertainty. After searching for the two LRs to register the manipulator to the RMMA, the manipulator can then infer the relative poses APs on the RMMA and therefore, the EOAT uncertainty analysis can be isolated from the mobile robot uncertainty.

\subsection{Measurement Uncertainty}

A comparison of the RMMA versus the OTS methods for measuring mobile manipulator performance was assessed. The EOAT's distance traversed between the RMMA targets derived from the manipulator log data and the OTS measurements over the ten trials were used to assess the two measurement methods.

There were four EOAT moves after registration as shown in Table 8, 1) from LR2 to AP1, 2) from AP1 to AP2, 3) from AP2 to AP1, and 4) from AP1 to AP2 again. There were two possible occurrences after each EOAT move to AP: 1) the EOAT detected the AP and logged the EOAT pose or 2) the EOAT failed to detect the AP and then began a square spiral 
search until the reflector was detected. For each move, Table 8 shows the actual distance based on the RMMA design specifications, the manipulator logged distance traversing between the targets, the EOAT distances tracked by the OTS, and the difference in the logged manipulator and OTS tracked distances of the EOAT. A measurement bias of about 2 $\mathrm{mm}$ was observed, along with a standard deviation of over $1 \mathrm{~mm}$. The measurement uncertainty using the RMMA was within $2 \mathrm{~mm}$ using the OTS as a reference.

Table 8. Mean and [standard deviations] of stop positions (in mm) at AP's for the manipulator as (A) logged from the manipulator control program, (B) measured by the OTS, and $(C)$ offset between the target distances derived from the manipulator log and the OTS measurements of the manipulator pause locations.

\begin{tabular}{|l|l|l|l|l|}
\hline $\begin{array}{l}\text { Manipulator } \\
\text { Move }\end{array}$ & $\begin{array}{l}\text { Actual } \\
\text { RMMA } \\
\text { distances }\end{array}$ & $\begin{array}{l}\text { (A) } \\
\text { manipulator } \\
\text { log }\end{array}$ & $\begin{array}{l}(C=|A-B|) \\
\text { manipulator } \\
\text { uncertainty }\end{array}$ \\
\hline LR2 --> AP1 & 460.01 & $459.24[0.38]$ & $460.53[1.03]$ & $1.42[0.75]$ \\
\hline AP1 --> AP2 & 457.20 & $455.92[0.74]$ & $457.56[0.98]$ & $1.83[1.00]$ \\
\hline AP2 --> AP1 & 457.20 & $455.73[0.88]$ & $457.47[1.06]$ & $1.82[1.04]$ \\
\hline AP1 --> AP2 & 457.20 & $455.37[1.15]$ & $457.06[1.13]$ & $1.78[0.93]$ \\
\hline
\end{tabular}

The number of spiral search steps and related statistics were also used to characterize the manipulator performance. Upon registration to the RMMA, the mobile manipulator was expected to move the EOAT directly from bisecting the two large reflectors (i.e., FinalEOAT for LR1 and LR2) to the two $2 \mathrm{~mm}$ diameter AP reflectors. If the APs were not immediately detected, the AP detection was deemed a failure and a $0.5 \mathrm{~mm}$ square step search was initiated to determine the error offset from the AP.

Table 9 shows the EOAT registration searches and search steps for the two APs at the two MBs. The search and search steps were derived from the manipulator's log file. No searches were needed if the manipulator immediately detected the AP. Otherwise, the number of search steps was based on the $x$ and $y$ changes logged during the square spiral search after the initial pause. A maximum of 375 search steps was required at AP1 when the mobile manipulator was positioned at MB1. At MB1, AP2 positions were detected relatively quickly mainly because the manipulator control algorithm was designed to correct the AP1to-AP2 EOAT maneuver by using the AP1 detection pose as an initial point to traverse the $457.2 \mathrm{~mm}$ to the AP2 pose. However, at MB2, it was observed that the manipulator required additional searches for AP2.

The additional searches required at MB2 may be due to the heading angle discrepancy observed. On average, the mobile robot's computed heading angle relative to the RMMA at MB2 and the OTS measured heading angle was greater than at MB1 (Table 6). The discrepancy in the mobile manipulator's position and heading angle could have contributed to the manipulator's perceived distance estimation error. 
Table 9. Mobile manipulator registration showing number of searches over the ten trials, the max and mean number search steps per search, distance from initial to final pose, and

search duration for APs.

\begin{tabular}{|l|l|l|l|l|l|}
\hline & & $\begin{array}{l}\text { \# of } \\
\text { searches } \\
\text { at AP }\end{array}$ & $\begin{array}{l}\text { Search Steps } \\
\text { (Max, Mean) } \\
\text { [Stdev], number } \\
\text { of steps }\end{array}$ & $\begin{array}{l}\text { Offset Distance } \\
\text { (Max, Mean) } \\
\text { [Stdev], mm }\end{array}$ & $\begin{array}{l}\text { Search } \\
\text { duration } \\
\text { (Max, Mean) } \\
\text { [Stdev], } s\end{array}$ \\
\hline \multirow{2}{*}{ MB1 } & AP1 & 6 & $375,90[148]$ & $5.59,2.22[2.03]$ & $20,5.16[7.65]$ \\
\cline { 2 - 6 } & AP2 & 0 & $0,0[0]$ & $0,0[0]$ & $0,0[0]$ \\
\hline \multirow{2}{*}{ MB2 } & AP1 & 0 & $0,0[0]$ & $0,0[0]$ & $0,0[0]$ \\
\cline { 2 - 6 } & AP2 & 2 & $68,47.5[29]$ & $2,1.75[0.35]$ & $1,1[0]$ \\
\hline $\begin{array}{l}\text { For all } \\
\text { (out of 80) }\end{array}$ & & 8 searched & $375,79.3[127.1]$ & $5.59,2.01[1.74]$ & $20,4.12[6.75]$ \\
\hline
\end{tabular}

There were several additional factors that contributed to system errors, such as: alignment errors between the mobile base and the manipulator base centroids, variability of the travel surface, alignment of RMMA fixtures, and the manipulator and the base stability during its search for the fiducial, resulting in different failure rates for each AP. As a comparison, data logged by the manipulator to determine registration success was corroborated with the OTS measurements to determine mobile manipulator registration performance.

\section{Discussion}

In this study, positioning precision and the uncertainty of the advanced mobile manipulator system was characterized in order to understand the key sources of error in the robot navigation, manipulator positioning, and in the measurement method. The experiment also provided insights of how to reduce the uncertainty in future operations (e.g., in fixtureless docking, marker placement, registration, and assembly). Given the greater variability in the environment and in the mobile manipulator system components, there remain inherent uncertainty factors of the individual components (mobile robot, support structures, manipulator, and tooling), which impact the performance of the integrated mobile manipulator system, and also the navigation, the manipulator, and the measurement uncertainties.

The uncertainty contributions observed during the experiment are summarized below. The description of each source of uncertainty includes the impact of the uncertainty on experiment, the analysis, and the results. It is important for industry to consider the sources and magnitudes of the uncertainty contributions when attempting to assess the performance of mobile manipulators and their criticality to the safety and performance of the manufacturing process' tolerances and constraints. 


\subsection{Measurement Method Uncertainty}

The mobile manipulator was not parallel with the RMMA. Positioning the manipulator directly parallel to the RMMA was a challenge. Since the manipulator was a cantilevered load on the mobile robot when the manipulator is extended over the RMMA, the manipulator can cause the entire payload structure to tilt. The mobile manipulator was unable to detect differences in tilt caused by the extended manipulator whereas the OTS can measure the differences in actual EOAT position relative to the RMMA ground truth reflector locations.

Imperfect RMMA fixture positions. Although the RMMA had a machined hole position tolerance of $0.25 \mathrm{~mm}$, the reflective targets added to the RMMA for the LRs and APs were held by $3 \mathrm{D}$ printed mounts with a looser tolerance that can cause the potential position error to be more than the machined surface tolerance. Therefore, the RMMA ground truth measured using the OTS may have had an offset from the actual LR and AP positions, further contributing to the search error shown in Table 8. Furthermore, the height of the targets varied as much as $9 \mathrm{~mm}$ between the two MB sides, which indicated the floor surface level differed at each side.

Data alignment. While NTP was used to synchronize the OTS and controller computers, the timestamp precision between the manipulator controller, the mobile robot controller, and the OTS computers logging the pose data was around $1 \mathrm{~s}$ due to timestamping delay variability between the detection event and the transmission of the data over two wireless hops to the controller for timestamping and logging at the application layer. Wireless transmission and operating system processes can cause variability in the timestamping precision. To mitigate the timestamping challenge, the data fusion algorithm used both time and pause detection based on EOAT movement to minimize possible time errors. Data alignment errors affect the accuracy of the target position estimation of the manipulator at the time of the measurement. Onboard data acquisition and timestamping of registration events at the hardware layer with a synchronized clock would improve data alignment of the mobile robot and the manipulator controller data with the OTS data.

During the experiments, all experimental factors were strictly controlled. For the data analysis, several assumptions were made. The first assumption was that the data can be readily converted from a $3 D$ environment to a $2 \mathrm{D}$ coordinate frame with negligible error contribution. Even though the OTS and the mobile manipulator captured 3D pose data, the mobile base used a 2D navigation system. In addition, the target assembly operation was based on a 2D flat surface. Possible errors from this assumption were OTS data conversion error, the ground surface and the RMMA were not parallel, and the manipulator support structure was not leveled relative to the mobile robot.

The second assumption was that the OTS is the reference or ground truth. As a highly proven technology for measuring and tracking rigid body poses, the OTS measurements were used as ground truth reference with a previously established uncertainty [21]. However, careful marker placement to minimize marker occlusion and marker ambiguities is important in ensuring optimal motion capture. Marker placement issues can lead to marker ambiguity and degrade the accuracy and uncertainty of the ground truth measurements. 
Finally, the third assumption was that the mobile robot stopped completely at each side of the RMMA, while the mobile manipulator operated. This study assumed that the mobile robot remained stationary relative to the RMMA throughout the simulated manipulator assembly task.

\subsection{Mobile Manipulator System Uncertainty}

Alignment of centroids of the mobile robot and manipulator were not coincident. The challenge of aligning the mobile robot and the manipulator, which were manufactured independently, was due to the integration of the components. The mobile base coordinate system was centered between the wheels and the mounting surface. Registering the manipulator to the mobile robot was therefore difficult to directly measure. Hence, the two references may not be coincident. The mobile manipulator positioning was impacted, because the commanded position of the mobile robot should be set slightly ahead of the LR/AP centers to stop between them. The error source can be corrected by determining the position and orientation offset. The offset was determined to be $x=0.35 \mathrm{~mm}, y=2.10 \mathrm{~mm}$, and $\theta=-7.82^{\circ}$. Accordingly, the poses of MBs were shifted to align the two components of the mobile manipulator. When converting the manipulator EOAT pose into the mobile base coordinate system, the converted poses were shifted according to the derived translation and rotation components of the transformation matrix. The commanded poses were shifted to the right at MB1 and to the left at MB2 based as depicted in Fig. and Fig. 6.

Manipulator stability. Stability challenges due to 1) the cantilevered manipulator over the RMMA, 2) the short wheelbase of the mobile robot, and 3) any manipulator motion at the parked mobile robot locations, would have caused the mobile manipulator to vibrate, especially when performing search operations. The force of the vibration could have changed the mobile robot pose during the manipulator search operations. A change in the mobile robot base position could potentially cause erroneous LR center-detection, an AP-detect failure, and the need for additional search time.

\section{Future Work}

For future work, we propose to improve upon the limitations in the data acquisition, including timestamping the registration events and location data onboard the mobile manipulator to minimize variability in data transmission latency. The clocks of the mobile manipulator loggers would be synchronized via wireless to a GPS-based time server which provides accuracy to UTC within $100 \mathrm{~ns}$. Additionally, we propose to make improvements to the test method to include a digital level in quantifying the surface floor angle variability by measuring the relative angle of the mobile manipulator as the system traverses through the simulated industrial environment. By quantifying the surface floor angles, the goal is to understand how surface level variations in the environment can contribute to the mobile robot base navigation and manipulator positioning uncertainty. While the RMMA enables a low-cost method to assess manipulator and navigation uncertainty, improvements in reducing the measurement uncertainty would enable performance testing of advanced mobile manipulators for higher precision industrial assembly tasks [27]. 


\section{Conclusion}

This paper described improvements in the test methodology and additional performance metrics to support the development of objective test methods for characterizing advanced mobile manipulators with a mobile robot base. One of the key challenges was the additional dynamic uncertainty sources introduced with the use of an advanced mobile robot base. Using the relatively inexpensive RMMA, as compared to an OTS, we demonstrated and validated a proposed test methodology for advanced mobile manipulators. A ground truth OTS system measured the RMMA target locations and then simultaneously measured the mobile robot and manipulator end-effector during the experiment. Logged position data from the mobile manipulator navigation base and the OTS had different coordinate reference frames, which required a registration method to align. Following, the manipulator and the mobile robot coordinate system were registered to the OTS coordinate system such that the logged data could be compared to the OTS data to determine the positioning offset of the mobile manipulator relative to the RMMA, and to assess the measurement bias and uncertainty between the logged data and the OTS.

The analysis quantified the error contributions of the main components of the advanced mobile manipulator system, the uncertainty of the mobile robot, EOAT, and the aggregate errors of the mobile manipulator and found the offset differences in all three sources compared to the OTS. The experimental results showed the logged position of the mobile robot differed from the OTS ground truth with a maximum of $35.7 \mathrm{~mm} \pm 5.4 \mathrm{~mm}$. Based on the empirical measurements, the maximum offset observed between the EOAT logged position and the OTS was $1.83 \mathrm{~mm} \pm 1.0 \mathrm{~mm}$. The key uncertainty factors in the experimental execution and data analysis included the offset of the centroid alignment between the mobile robot and the manipulator, mobile robot alignment error to the RMMA, RMMA fixture position errors, mobile manipulator stability, marker placement, and temporal alignment of the data. The study included an analysis of the sources of the mobile manipulator and measurement error and their impact on the mobile manipulator performance and measurement uncertainty.

The experiment and analysis demonstrated how a low-cost and readily reconfigurable RMMA test method, can be applied to other mobile manipulators using the authors' systemunder-test, with a measurement uncertainty of less than $2 \mathrm{~mm}$. Because the RMMA targets had a diameter of $2 \mathrm{~mm}$, the resulting uncertainty was acceptable. The experiment validated the RMMA measurement method using the mobile base and manipulator controller logs and compared the logged measurements with the OTS measurements. Additionally, the measurement method using the RMMA and verified with OTS measurements, as modeled in [18], could be applied in-situ for mobile manipulator performance measurement during manufacturing. The results from the study indicate there may be additional parameters contributing to a manipulator's positioning precision due to properties of individual system components and environmental conditions on the performance of advanced mobile manipulator systems. Finally, through the application of the RMMA test artifact, the implementation of time synchronization and fusion of disparate data sources including an example mobile robot base, an OTS ground truth measurement system, an onboard manipulator, and a method for coordinate registration from the mobile manipulator logged position data to the OTS, we have demonstrated a proposed method to quantify the impact of several error sources. 


\section{Acknowledgments}

We would like to thank Tsai Hong for providing guidance on using the OTS system and software and on working with the OTS data.

\section{References}

[1] Martínez-Barberá, H. and Herrero-Pérez, D., "Autonomous navigation of an automated guided vehicle in industrial environments." Robotics and Computer-Integrated Manufacturing, 2010, pp.296-311.

[2] Durrant-Whyte, H.F., "An autonomous guided vehicle for cargo handling applications." The International Journal of Robotics Research, 1996, pp.407-440.

[3] Hamner, B., Koterba, S., Shi, J., Simmons, R., and Singh, S., "An autonomous mobile manipulator for assembly tasks." Autonomous Robot, 2010, pp.131-149.

[4] Bøgh, S., Hvilshøj, M., Kristiansen, M., and Madsen, O., "Autonomous industrial mobile manipulation (AIMM): from research to industry." In 42nd International Symposium on Robotics, 2011, pp.1-9.

[5] Bostelman, R., Hong, T., and Marvel, J., "Survey of Research for Performance Measurement of Mobile Manipulators," Journal of Research of the National Institute of Standards and Technology, Volume 121 (2016), http://dx.doi.org/10.6028/jres.121.015.

[6] Bostelman, R. "Performance measurement of mobile manipulators." Robotics [cs.RO]. PhD Thesis, Université Bourgogne Franche-Comté, 2018.

[7] Seelinger, M., Yoder, J.D., Baumgartner, E.T., and Skaar, S.B., "High-precision visual control of mobile manipulators." IEEE Transactions on Robotics and Automation, 18(6), 2002, pp.957-965.

[8] Khatib, O., et al. "Coordination and decentralized cooperation of multiple mobile manipulators." Journal of Robotic Systems 13(11), 1996, pp.755-764.

[9] Papadopoulos, E.G., and Rey, D.A., "A new measure of tipover stability margin for mobile manipulators." Robotics and Automation, 1996. Proceedings., 1996 IEEE International Conference on. Vol. 4. IEEE, 1996.

[10] Dömel, A., Kriegel, S., Kaßecker, M., Brucker, M., Bodenmüller, T., and Suppa, M., 2017. "Toward fully autonomous mobile manipulation for industrial environments." International Journal of Advanced Robotic Systems, 14(4), pp.1-19.

[11] Shneier, M. and Bostelman, R., "Literature Review of Mobile Robots for Manufacturing.” NIST Internal Report \#8022, 2014.

[12] "Yaskawa Motoman MH80 robot unloading trucks - from Wynright Corporation," http://www.youtube.com/watch?v=8wngL0BnF_4, June 18, 2013.

[13] Guizzo, E., "Meka Robotics, Announces Mobile Manipulator With Kinect and ROS," http://spectrum.ieee.org/automaton/robotics/humanoids/meka-robotics-announcesmobile-manipulator-with-kinect-and-ros, 16 Feb 2011. 
[14] Green, T., “KUKA Falls First, Buys Swisslog for \$335M. Who’s Next?” Robotics Business Review, Sept 29, 2014.

[15] NIST Robotic Systems for Smart Manufacturing Program, https://www.nist.gov/programs-projects/measurement-science-manufacturing-robotics, accessed January 7, 2020.

[16] Bostelman, R., Hong, T., Legowik, S., and Shah, M., "Dynamic Metrology and ASTM E57.02 Dynamic Measurement Standard." Journal of the CMSC, Vol. 12, No. 1, Spring 2017.

[17] Bostelman, R., Hong, T., and Marvel, J., "Performance Measurement of Mobile Manipulators.” SPIE 2015, Baltimore, MD, April 2015.

[18] Bostelman, R., Foufou, S., Hong, T., and Shah, M., "Model of Mobile Manipulator Performance Measurement using SysML." Journal of Intelligent \& Robotic Systems, 2017, pp.1-19.

[19] The Network Time Protocol (NTP) Distribution, https://www.eecis.udel.edu/ mills/ntp/ html/index.html, accessed December 3, 2017.

[20] Support boundary to configure the Windows Time service for high-accuracy requirements, https://support.microsoft.com/en-us/help/939322/support-boundary-toconfigure-the-windows-time-service-for-high-accura, accessed December 3, 2017.

[21] Bostelman, R., Falco, J., and Hong, T., "Performance Measurements of Motion Capture Systems used for AGV and Robot Arm Evaluation." Autonomous Industrial Vehicles: From the Laboratory to the Factory Floor, STP1594, Book Chapter 7, May 2016.

[22] Shah, M., Bostelman, R., Legowik, S., and Hong, T., "Calibration of mobile manipulators using 2D positional features.” Measurement, 124, 2018, pp.322-328.

[23] Optitrack Motive Calibration Documentation. https://v21.wiki.optitrack.com/index.php?title=Calibration. Accessed December 4, 2019.

[24] Universal Robots UR5 Documentation. https://www.universalrobots.com/media/50588/ur5 en.pdf. Accessed October 29, 2019.

[25] Shah, M., "Comparing Two Sets of Corresponding Six Degree of Freedom Data." Computer Vision and Image Understanding, 115(10), 2011, pp.1355-1362.

[26] Bostelman, R. Eastman, R., Hong, T., Aboul-Enein, O., Legowik, S., and Foufou, S. "Comparison of registration methods for mobile manipulators." Advances in Cooperative Robotics, 2017, pp.205-213.

[27] Inoue, T., De Magistris, G., Munawar, A., Yokoya, T. and Tachibana, R. “Deep reinforcement learning for high precision assembly tasks." 2017 IEEE/RSJ International Conference on Intelligent Robots and Systems (IROS), September 2017, pp.819-825. 